\title{
Characterization of atherosclerotic carotid plaque using MATCH: initial clinical experience
}

\author{
Wei Yu ${ }^{1 *}$, Zhaoyang Fan², Lixin Yang ${ }^{1}$, Yibin Xie ${ }^{2,3}$, Li Dong ${ }^{1}$, Zhanhong Wang ${ }^{1}$, Yanni $\mathrm{Du}^{1}$, Xiaoming $\mathrm{Bi}^{4}$, Jing $\mathrm{An}^{5}$, \\ Tianjing Zhang ${ }^{5}$, Gerhard Laub ${ }^{4}$, Zhaoqi Zhang ${ }^{1}$, Debiao Li $i^{2,3}$ \\ From 18th Annual SCMR Scientific Sessions \\ Nice, France. 4-7 February 2015
}

\section{Background}

Characterization of carotid artery morphology and plaque composition requires multiple measurements with different contrast-weightings, which are limited by long scan time, image misregistration, and expertise-dependency in image interpretation. Recently, a new method,3D Multicontrast ATherosclerosis CHaracterization (MATCH) has been developed to overcome the above drawbacks by acquiring multi-contrast weighted images in one scan [1] This study was conducted to determine the accuracy of MATCH in the characterization of plaque morphology and composition in patients.

\section{Methods}

Thirty patients scheduled for carotid revascularization underwent preoperative carotid MRI with MATCH and the conventional multi-contrast protocol (T1W, T2W, TOF,CE-T1W) in the same examination with a 3T scanner(Table 1) and 8-channel carotid coil. All image sets were processed using plaque analysis software (MRI-Plaque View, VPDiagnostics). Blinded image review for anatomy and composition identification was performed by 2 radiologists (with 2 and 9-year experience in carotid plaque MR characterization). Images from each artery underwent location matching process (including image reformation in 3D TOF) to account for inconsistency in slice number and thickness between the two protocols and inter-scan motion. Quantitative area measurements of the lumen and wall of the bilateral carotid arteries were obtained from T2-w images. The normalized wall index (NWI) was calculated by dividing the wall area by the total vessel area (lumen+wall). The presence of intraplaque hemorrhage (IPH), calcification

'Department of Radiology, Beijing anzhen Hospital, Capital Medical University, Beijing, China

Full list of author information is available at the end of the article
(CA), and lipid-rich necrotic core (LRNC) were determined using the criteria for the MATCH protocol [1] and those in a review article for the conventional protocol [2] The paired t-test was used to compare the two measurements. Cohen kappa (K) was computed to quantify the agreement in the detection of components between the two protocols.

\section{Results}

The two protocols were successfully performed in all 30 patients. The scan time was about $15 \mathrm{~min}$ for the conventional protocol and $5 \mathrm{~min}$ for the MATCH protocol. The 30 patients bilateral artery yielded 898 matched MRI cross-sectional slices for analysis. The statistical analysis shown no significant difference of mean area of lumen ( 36.27 vs $\left.35.72 \mathrm{~mm}^{2}, \mathrm{p}=0.62\right)$, wall (42.80 vs $\left.43.64 \mathrm{~mm}^{2}, \mathrm{p}=0.82\right)$ and NWI (59.21 vs 59.81, $\mathrm{p}=0.72$ ) measured by MATCH and conventional protocols. Moderate to good agreement was seen between 2 protocols in the detection of plaque components (LR/NC $\mathrm{k}=0.84$, CA $\mathrm{k}=0.802$, IH k=0.773).

\section{Conclusions}

Our preliminary clinical study suggests that MATCH has similar performance in the evaluation of carotid plaque to the conventional multi-contrast protocol. Shorter scan time, less image misregistration and relatively simplified criteria for component have substantially reduced the examination failure rate and shorten the time consuming of interpretation. $\mathrm{MATCH}$ is a promising CMR imaging method for assessing the risk of plaque disruption in a clinical workup.

\section{Funding}

Beijing health system of high-level technical personnel training project 2013-2-005. 
Table 1 Scan Parameters of Conventional and MATCH Protocol

\begin{tabular}{cccccc}
\hline & TOF & T1W /TSE & T2W TSE & CE-T1W & MATCH \\
\hline TE/TR (ms) & $3.6 / 21$ & $12.0 / 800$ & $59.0 / 4000$ & $12.0 / 800$ & $4.2 / 10.8$ \\
\hline In-plane resolution (mm2) & $0.87 \times 0.52$ & $0.55 / 0.63 \times 0.55 / 0.63$ & $0.55 / 0.63 \times 0.55 / 0.63$ & $0.55 / 0.63 \times 0.55 / 0.63$ & $0.55 / 0.63 \times 0.55 / 0.63$ \\
\hline Number of slices & 84 & 18 & 16 & 16 & 16 \\
\hline Slice thickness (mm) & 1 & 2 & 2 & 2 & 2 \\
\hline Number of Average & 1 & 2 & $120-160$ & $136-170$ & 7 \\
\hline Flip angle & 25 & $136-170$ & 12 & 407 & $55-67$ \\
\hline Echo train length & - & 7 & 407 & $2: 56$ & 130 \\
\hline Bandwidth (MHz) & 250 & 407 & $3: 24$ & $4: 45$ & \\
\hline Scan time (min:sec) & $2: 27$ & $2: 56$ & & & 2 \\
\hline
\end{tabular}

\section{Authors' details}

'Department of Radiology, Beijing anzhen Hospital, Capital Medical

University, Beijing, China. ${ }^{2}$ Biomedical Imaging Research Institute, CedarsSinai Medical Center, Los Angeles, CA, USA. ${ }^{3}$ Department of Bioengineering, University of California, Los Angeles, CA, USA. ${ }^{4}$ MR R\&D, Siemens Healthcare, Los Angeles, CA, USA. ${ }^{5}$ MR Collaborations NE Asia, Siemens Healthcare, Beijing, China.

Published: 3 February 2015

doi:10.1186/1532-429X-17-S1-072

Cite this article as: Yu et al:: Characterization of atherosclerotic carotid plaque using MATCH: initial clinical experience. Journal of Cardiovascular

Magnetic Resonance 2015 17(Suppl 1):O72.

Submit your next manuscript to BioMed Central and take full advantage of:

- Convenient online submission

- Thorough peer review

- No space constraints or color figure charges

- Immediate publication on acceptance

- Inclusion in PubMed, CAS, Scopus and Google Scholar

- Research which is freely available for redistribution

Submit your manuscript at www.biomedcentral.com/submit
C Biomed Central 